\title{
Continuum Resonances with Shielded Coulomb-Like Potential and Efimov Effect*
}

\author{
D. S. Tusnski ${ }^{1 * *}$, M. T. Yamashita ${ }^{1 * * *}$, T. Frederico ${ }^{2)}$, and L. Tomio ${ }^{1), 3)}$ \\ Received January 29, 2013
}

\begin{abstract}
Motivated by the possibility of the second energy level $\left(0_{2}^{+}\right)$of ${ }^{12} \mathrm{C}$ (in a three-alpha model) to turn into an Efimov state, we study a simple non-realistic toy model formed by three bosons interacting by the phenomenological $s$-wave Ali-Bodmer potential plus a Coulomb interaction. An artificial three-body potential was used to create a resonance with energy close to the energy of the $0_{2}^{+}$of ${ }^{12} \mathrm{C}, 0.38 \mathrm{MeV}$. The strength of the Coulomb potential is decreased until the energies of the two alpha pairs are zero. The system was placed inside a harmonic trap and a stabilization method has been used to calculate the energies of the resonances. We found that the shielded-Coulomb potential, which keeps the long tail, is not able to produce the Efimov effect. The energy of the three alphas decreases only to $0.19 \mathrm{MeV}$ when the two-body energy crosses the threshold to become bound.
\end{abstract}

DOI: $10.1134 / \mathrm{S} 1063778814040152$

We cordially dedicate this work to Vladimir B. Belyaev on the occasion of his 80th birthday

\section{INTRODUCTION}

The theoretical prediction of Efimov states in 1970 [1] was made in the context of nuclear physics. However, the experimental achievement of this counterintuitive phenomenon has been made in an ultracold gas of cesium atoms [2]. Since then, once realized that such effect is not a simple mathematical artifact emerging in the limit of zero two-body binding, lot of papers have been published in this topic, starting a new branch of quantum few-body physics, called "Efimov Physics" [3].

Originally, the Efimov effect was derived for a system of three spinless neutral particles of equal mass and their possible implications have been considered for the nuclei ${ }^{12} \mathrm{C}$ and ${ }^{3} \mathrm{H}[1]$. This effect is manifested by the appearance of an infinite number of three-body bound states as the two-body energy tends to zero, or equivalently the two-body scattering length tends to infinity, which can be explained by the

\footnotetext{
* The text was submitted by the authors in English.

${ }^{1)}$ Instituto de Física Teórica, UNESP-Universidade Estadual Paulista, São Paulo, Brazil.

${ }^{2)}$ Instituto Tecnológico de Aeronáutica-DCTA, S. J. dos Campos, Brazil.

${ }^{3)}$ Universidade Federal do ABC, Santo André, Brazil.

${ }^{* *}$ E-mail: saracol@ift.unesp.br

${ }^{* * *}$ E-mail: yamashita@ift.unesp.br
}

appearance of an effective potential proportional to $1 / R^{2}\left(R^{2}=r_{12}^{2}+r_{13}^{2}+r_{23}^{2}\right.$, where $r_{i j}$ is the distance between particles $i j$ ) [4]. The energy ratio between two consecutive states is given by $e^{2 \pi / s_{0}}$, where the constant $s_{0}$ depends on the mass ratio of the particles (for equal masses $s_{0} \approx 1.00624$ ). Their rootmean-square radii, $\sqrt{\left\langle R^{2}\right\rangle}$, also present a constant ratio between two consecutive states given by $e^{\pi / s_{0}}$. Nowadays, we know that this effect is possible for several systems with different masses and also for more than three particles [5].

Efimov states are very weakly bound systems, their wave functions are extremely extended in space and located outside the range of the potential, such that their properties are independent of the details of the short-range potential [6]. Obviously, these characteristics cannot be expected to appear in a deeply bound and compact nuclear system such as the ${ }^{12} \mathrm{C}$ ground state. The ${ }^{12} \mathrm{C}$, approximated by three alpha particles, also presents a Coulomb interaction, such that the collapse of the system, known as Thomas collapse [7], is avoided (note that the appearance of Efimov effect is closely related to the Thomas collapse [8]). Moreover, ${ }^{8} \mathrm{Be}$ does not have zero energy and we do not have the freedom, as in atomic systems, to manipulate the two-body energy. These reasons might be enough to exclude the possibility to observe the Efimov effect in such non-exotic nuclei ${ }^{4)}$.

\footnotetext{
${ }^{4)}$ For a recent report on alpha cluster description of nuclei, see [9]. In particular, the treatment of three-body charged particles is discussed by Belyaev and collaborators in [10].
} 
As exposed in the last paragraph, the freedom we have in atomic condensates to manipulate the twobody scattering length by using the Feshbach resonance [11] is not present in nuclei. However, the Coulomb potential could eventually be shielded in a plasma with an electronic density enough to decrease the energies of ${ }^{12} \mathrm{C}$ and ${ }^{8} \mathrm{Be}[12]$, such that Higa et al. conjectured that "the ${ }^{12} \mathrm{C}$ Hoyle state is a remnant of a Efimov state that appears in the unitary limit" [13]. In this paper, we use a very simple nonrealistic toy model to make a first investigation of the former conjecture. We study a system of three bosons (3B) in an $s$-wave state, where the two bosons (2B) interact by the phenomenological $s$-wave AliBodmer potential [14]. This is a very preliminary calculation which may give a first insight about the conjecture. The paper is organized as follows. In Section 2, we detail the method used to calculate the resonances. In Section 3 we present our results. The conclusions are presented in Section 4.

\section{FORMALISM}

We calculated a three-boson system with total angular momentum zero. For the two-body interaction we used the phenomenological $s$-wave Ali-Bodmer potential given by

$$
V\left(r_{i j}\right)=500 e^{-\left(0.7 r_{i j}\right)^{2}}-130 e^{-\left(0.475 r_{i j}\right)^{2}},
$$

where $r_{i j}$ is the distance between the bosons $i$ and $j$. All energies and distances are given in $\mathrm{MeV}$ and $\mathrm{fm}$.

We also included the following three-body potential to create artificially a resonance close to the experimental energy of $0.38 \mathrm{MeV}$ for the $0_{2}^{+}$state

$$
V_{3}(R)=-9.02 e^{-(R / 6)^{2}},
$$

where $R \equiv \sqrt{r_{12}^{2}+r_{23}^{2}+r_{31}^{2}}$. We should mention here that the two-body potential used in this toy model does not consider higher partial waves, in particular $d$ and $g$ waves, which give a significant contribution to the properties of ${ }^{12} \mathrm{C}$ [15]. Much more realistic calculations can be found in $[16,17]$.

The energy of the resonances was calculated by using a stabilization method $[18,19]$. For this purpose, the system was put inside a harmonic trap

$$
V_{\text {trap }}\left(r_{i}\right)=\frac{\hbar^{2}}{2 m} \frac{r_{i}^{2}}{b^{4}}
$$

where $m$ is the mass of the alpha particle and $r_{i}$ are the single-boson coordinates. The size of the trap, $b$, is varied and the resonance appears as a "plateau" revealed by the discretized-continuum states, which present the noncrossing behavior at the resonance energy.

PHYSICS OF ATOMIC NUCLEI Vol.77 No. 42014
The eigenvalues are approached by $E \equiv \frac{\langle\psi|H| \psi\rangle}{\langle\psi \mid \psi\rangle}$, where $H$ is the Hamiltonian of the system and the wave function was expanded by a sum given by

$$
|\psi\rangle=\sum_{i=1}^{N} C_{i} S\left|\phi_{i}\right\rangle,
$$

where $N$ is the number of terms of the basis, large enough to have a converged value for $E$, and $S$ is a symmetrizer operator. The functions $\phi_{i}$ have been chosen as correlated Gaussians [20, 21]

$$
\phi_{i}=\exp \left[-\frac{1}{2} \sum_{j>k=1}^{N_{P}} \alpha_{j k}^{(i)}\left(\mathbf{r}_{j}-\mathbf{r}_{k}\right)^{2}\right],
$$

where $N_{P}$ is the number of bosons, $\left\{\mathbf{r}_{1}, \mathbf{r}_{2}, \ldots, \mathbf{r}_{N_{P}}\right\}$ are the single-boson coordinates and $\alpha_{j k}^{(i)}$ is a parameter related to the extension of the wave function of each pair $j k$.

Equation (5) can be written in terms of the Jacobi coordinates as follows. The relative coordinates, given by $\left\{\mathbf{x}_{1}, \mathbf{x}_{2}, \ldots, \mathbf{x}_{N_{P}-1}\right\}\left(\mathbf{x}_{N_{P}}\right.$ is chosen as the center-of-mass coordinate), can be written in terms of the single boson coordinates by using a transformation matrix $U$ :

$$
\mathbf{x}_{i}=\sum_{j=1}^{N_{P}} U_{i j} \mathbf{r}_{i}
$$

such that the functions $\phi_{i}$ can now be written in a compact notation as

$$
\phi_{i}(\mathbf{x})=\exp \left[-\frac{1}{2} \tilde{\mathbf{X}} A^{(i)} \mathbf{X}\right]
$$

where $\tilde{\mathbf{X}}$ is a row matrix with the relative coordinates and $A^{(i)}$ is a symmetric positive-definite $\left(N_{P}-1\right) \times$ $\left(N_{P}-1\right)$ matrix containing the $\alpha$ parameters to be defined conveniently. The success to obtain a good result for $E$ is very closely related to the choice of the matrices $A^{(i)}$ which means the parameters $\alpha_{j k}^{(i)}(j>$ $\left.k=1, \ldots, N_{P}\right)$. This choice can be made, for example, using a stochastic search, where the matrices are sorted in a certain interval. Another way is by using a geometric progression in which the parameters are written as $\alpha_{j k}^{(i)}=1 /\left(b_{0}^{j k} p^{i-1}\right)^{2}(i=1, \ldots, N)$, with $b_{0}$ and $p$ chosen to give a good description of the wave function.

The energy is then determined by solving the generalized eigenvalue problem,

$$
\sum_{j=1}^{N} H_{i j} C_{j}=E \sum_{j=1}^{N} b_{i j} C_{j}
$$



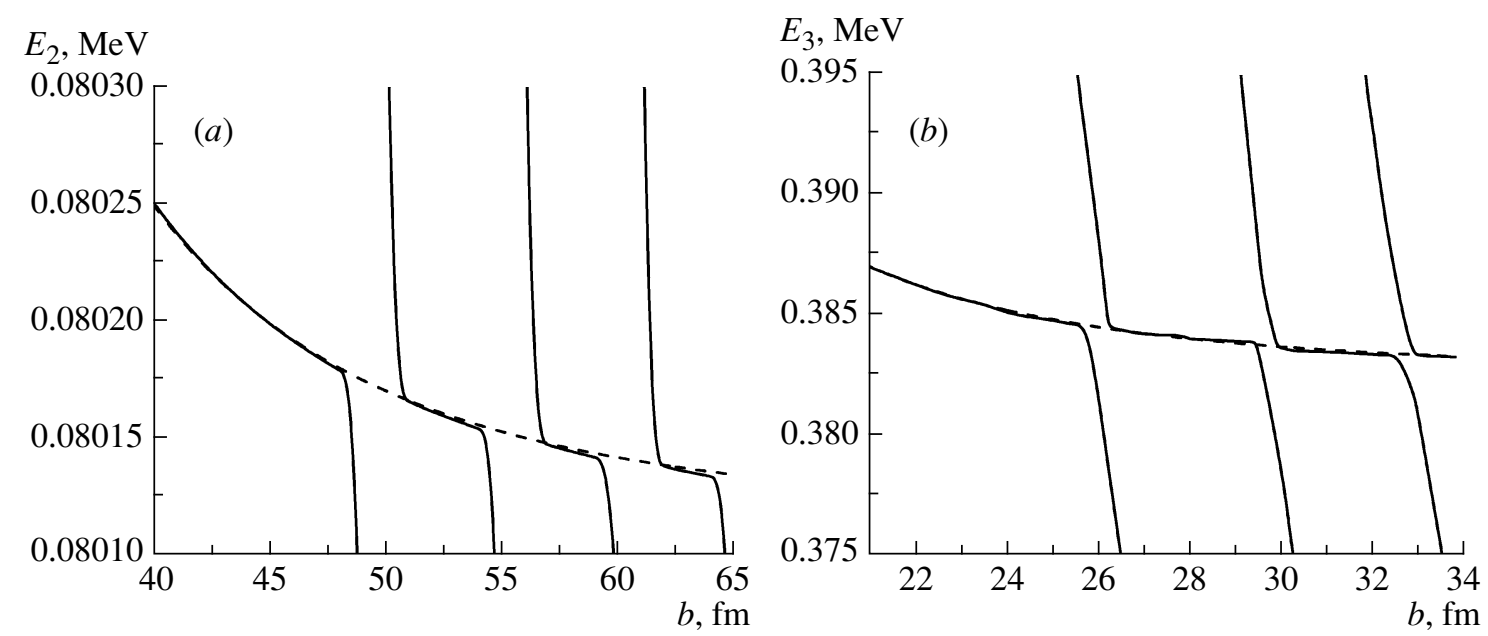

Fig. 1. Eingenvalues (solid lines) for two $(a)$ and three bosons $(b)$ as a function of the trap size, $b$. Both spectra present the avoid crossings close to the resonance energies. The parameters $\left(E_{r}, K\right)$ of the fitted function $E(b)=E_{r}+K b^{-4}$ are given by $(0.080,341.10)$ and $(0.38,856.72)$, respectively, for the two- and three-body systems (dashed lines).
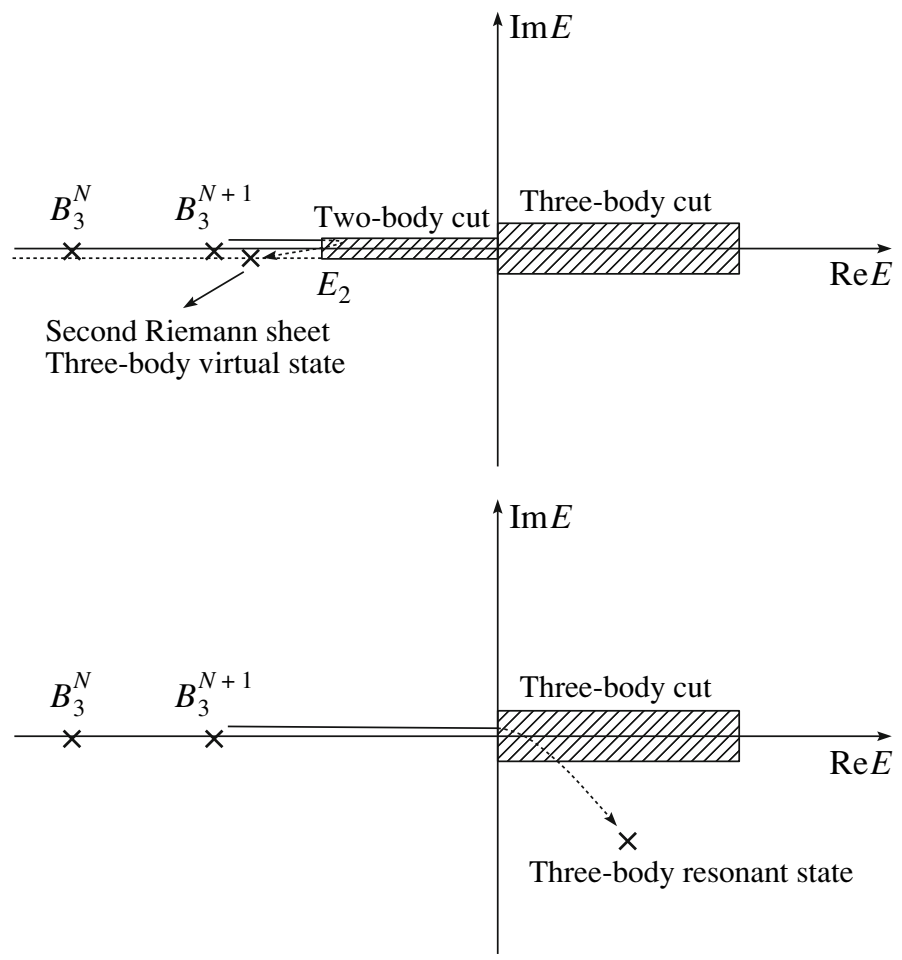

Fig. 2. Schematic representation of the analytical cut structure for a three-particle system. The upper diagram shows on the left side of the vertical axis the two-body cut defined by the two-body bound-state energy, $E_{2}=-\left|E_{2}\right|$, and the movement of the energy of an excited three-body bound state $B_{3}^{N+1}$ when $\left|E_{2}\right|$ is increased. In this case, the bound state becomes virtual, with the corresponding pole, located in the positive imaginary axis of the momentum plane, moving to the negative imaginary axis. In the lower frame the two-body system is virtual (no two-body cut) and the three-body state evolves into a resonance.

where $i=1, \ldots, N$ and the Hamiltonian and overlap matrices are given by

$$
H_{i j}=\left\langle S \phi_{i}|H| S \phi_{j}\right\rangle, \quad b_{i j}=\left\langle S \phi_{i} \mid S \phi_{j}\right\rangle .
$$

The evaluation of the matrix elements is facilitated because the mean value of the kinetic energy as well as the overlap and the potential can be calculated analytically since $\phi_{i}$ has been chosen as a Gaussian function.

The results for $E_{3}$ and $E_{2}$ are presented in Fig. 1. 


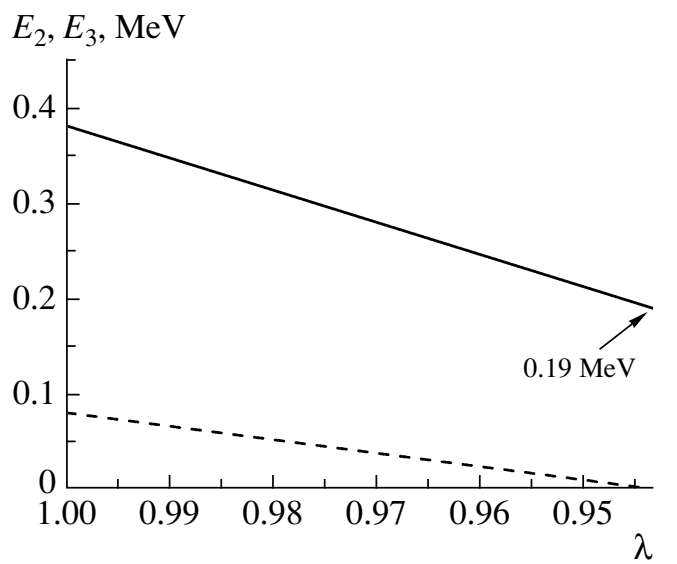

Fig. 3. Two- and three-boson energies (real part) as a function of $\lambda$. For $\lambda=0.943 E_{2}$ is zero and $E_{3}=$ $0.19 \mathrm{MeV}$.

For large $b$ we expect to fit a curve like $E(b)=E_{r}+$ $K b^{-4}$, where $E_{r}$ is the real part of the resonance energy [18].

\section{RESULTS}

The detailed pattern of the full trajectory of Efimov states is well known and has been studied in several papers [3, 22-24]. The upper part of Fig. 2 represents the S-matrix poles corresponding to two consecutive three-body bound states $\left(B_{3}^{N}\right.$ and $B_{3}^{N+1}$ ) and the disappearance of an excited state when the modulus of the two-body binding energy $\left(E_{2}\right)$ is increased. In this situation the $\mathrm{S}$-matrix bound-state pole becomes a virtual state passing through the elastic scattering cut with branch point at $E_{2}$. In the lower part of the figure, the two-body cut is absent for a virtual two-body state. In this case, the bound-state pole dives in the three-body continuum becoming a resonance [24]. The same qualitative behavior repeats for an $A A B$ system formed by two identical particles and one different [25].

The above-described behavior (lower part) would repeat in our $3 \mathrm{~B}$ case, when $E_{2}$ reaches the threshold to become a bound state, as a consequence of a greater attraction due to the shielding of the Coulomb. However, the condition for a $2 \mathrm{~B}$ system with zero energy still presents a repulsive barrier which should not be strong enough to prevent the collapse of the 3B system. The Coulomb potential $V_{\mathrm{C}}(r)=\lambda \frac{4 e^{2}}{r}$ is weakened by decreasing $\lambda$. Figure 3 shows the two- and three-body energies as a function of $\lambda$.

The three-body potential in the 3B system was used to set the energy close to the $0_{2}^{+}$state of ${ }^{12} \mathrm{C}$, $0.38 \mathrm{MeV}$. The $2 \mathrm{~B}$ system reaches the zero energy for $\lambda=0.943$. This critical $\lambda$ gives an energy of $0.19 \mathrm{MeV}$ for $3 \mathrm{~B}$. We would have obtained a similar effect for the decrease of the Coulomb potential by making it screened by electrons. In this case, the Coulomb is multiplied by $e^{-(r / \rho)^{2}}$ and the density of electrons involving the alpha particles can be directly associated with $\rho$. However, one should realize the mathematical difference between the screening keeping the Coulomb tail or by an exponential damping. In the last case, the long-range Efimov potential, when the two-body energy vanishes, can be active for distances larger than screening length, $\rho$, and weakly bound Efimov states with size much larger than $\rho$ can still appear.

\section{CONCLUSIONS}

This very preliminary calculation shows that the remaining repulsive barrier is still strong enough to prevent the Thomas collapse [7], and consequently the appearance of Efimov effect, of the $3 \mathrm{~B}$ system. The $3 \mathrm{~B}$ state will present a continuous transition to a bound state only for $\lambda=0.884$. Our results corroborate a previous calculation for ${ }^{12} \mathrm{C}$ made by Jensen et al. [12]. It is also worthwhile to mention another way to investigate the Efimov effect in the presence of a Coulomb potential; more specifically, by considering a zero-range potential for particles interacting via the Coulomb potential as done in [26].

This means that, besides, the resonant nature of the two-body subsystem, the three-body resonance does not turn into an Efimov state as conjectured in [13]. Obviously, a more detailed calculation should still be made in order to verify the extension of our results, as the two-body potential used in this toy model is not including higher partial waves, which are crucial to describe correctly the properties of ${ }^{12} \mathrm{C}[15-$ 17]. A detailed analysis of the structure of the Hoyle state in ${ }^{12} \mathrm{C}$, based on precise electron scattering data, can be found in [27].

\section{ACKNOWLEDGMENTS}

The authors thank the Brazilian agencies FAPESP and CNPq for partial support. We also would like to thank Prof. Dmitri Fedorov and Prof. A.S. Jensen for valuable suggestions.

\section{REFERENCES}

1. V. Efimov, Phys. Lett. B 33, 563 (1970).

2. T. Kraemer, M. Mark, P. Waldburger, et al., Nature 440,315(2006). 
3. M. T. Yamashita, D. V. Fedorov, and A. S. Jensen, Few-Body Syst. 51, 135 (2011); T. Frederico, L. Tomio, A. Delfino, M. R. Hadizadeh, and M. T. Yamashita, Few-Body Syst. 51, 87 (2011); T. Frederico, A. Delfino, L. Tomio, and M. T. Yamashita, Prog. Part. Nucl. Phys. 67, 939 (2012).

4. A. C. Fonseca, E. F. Redish, and P. E. Shanley, Nucl. Phys. A 320, 273 (1979).

5. E. Nielsen, D. V. Fedorov, A. S. Jensen, and E. Garrido, Phys. Rep. 347, 373 (2001); M. T. Yamashita, L. Tomio, A. Delfino, and T. Frederico, Europhys. Lett. 75, 555 (2006); Y. Castin, C. Mora, and L. Pricoupenko, Phys. Rev. Lett. 105, 223201 (2010); M. R. Hadizadeh, M. T. Yamashita, L. Tomio, A. Delfino, and T. Frederico, Phys. Rev. Lett. 107, 135304 (2011).

6. E. Braaten and H.-W. Hammer, Phys. Rep. 428, 259 (2006).

7. L. H. Thomas, Phys. Rev. 47, 903 (1935).

8. S. K. Adhikari, A. Delfino, T. Frederico, I. D. Goldman, and L. Tomio, Phys. Rev. A 37, 3666 (1988).

9. S. A. Sofianos, R. M. Adam, and V. B. Belyaev, Phys. Rev. C 84, 064304 (2011).

10. V. B. Belyaev and A. A. Naumkin, Few-Body Syst. 50, 403 (2011); V. B. Belyaev and I. I. Shlyk, Nucl. Phys. A 790, 792C (2007).

11. C. Chin, R. Grimm, P. Julienne, and E. Tiesinga, Rev. Mod. Phys. 82, 1225 (2010).

12. A. S. Jensen, D. V. Fedorov, K. Langanke, and H.-M. Müller, in Proceedings of the International Conference on Exotic Nuclei and Atomic Masses (ENAM-95), Ed. by M. de Saint Simon and O. Sorlin (Ed. Frontieres, Gif-sur-Yvette, 1995), p. 677.
13. R. Higa, H.-W. Hammer, and U. van Kolck, Nucl. Phys. A 809, 171 (2008).

14. S. Ali and A. R. Bodmer, Nucl. Phys. 80, 99 (1966).

15. I. Filikhin, V. M. Suslov, and B. Vlahovic, J. Phys. G 31, 1207 (2005).

16. R. Álvarez-Rodríguez, E. Garrido, A. S. Jensen, et al., Eur. Phys. J. A 31, 303 (2007).

17. S. I. Fedotov, O. I. Kartavtsev, and A. V. Malykh, JETP Lett. 92, 647 (2010).

18. D. V. Fedorov, A. S. Jensen, M. Thøgersen, et al., Few-Body Syst. 45, 191 (2009).

19. C. H. Maier, L. S. Cederbaum, and W. Domcke, J. Phys. B 13, L119 (1980).

20. Y. Suzuki and K. Varga, Stochastic Variational Approach to Quantum-Mechanical Few-Body Problems (Springer, Berlin, 1998).

21. Y. Suzuki and M. Takahashi, Phys. Rev. C 65, 064318 (2002).

22. E. A. Kolganova and A. K. Motovilov, Phys. At. Nucl. 62, 1179 (1999).

23. M. T. Yamashita, T. Frederico, and L. Tomio, FewBody Syst. 44, 191 (2008).

24. D. S. Tusnski, M. T. Yamashita, T. Frederico, and L. Tomio, Few-Body Syst. 54, 551 (2013).

25. M. T. Yamashita, T. Frederico, and L. Tomio, Phys. Lett. B 660, 339 (2008).

26. S. L. Yakovlev, C.-Y. Hu, and D. Caballero, J. Phys. B 40, 1675 (2007); S. L. Yakovlev and V. A. Gradusov, arXiv: 1205.5963 [physics.atom-ph].

27. M. Chernykh, H. Feldmeier, T. Neff, et al., Phys. Rev. Lett. 98, 032501 (2007); Phys. Rev. Lett. 105, 022501 (2010). 